\title{
DEVELOPMENT OF A FLEX-PLI SYSTEM MODEL AND INVESTIGATIONS OF INJURY
}

\author{
Yong-Ha Han ${ }^{1}$ In-Hyeok Lee ${ }^{2}$ Whe-Ro Lee \\ ${ }^{1}$ Hyundai Motor Company, Korea, yongha@hyundai . com \\ ${ }^{2}$ ESI Korea, Korea, \{inhyeok. lee, whe-ro.lee\} desi-group.com
}

\begin{abstract}
Pedestrian accidents give direct damage to the human body. Pedestrians do not have any safety devices and it results in a significant risk of injury to the pedestrians as compared to other accidents (Carroll, 2014). To protect pedestrians, EURO NCAP, JNCAP, and various pedestrian safety laws are enforced. Korea also imposes KNCAP and related laws. Assessment of pedestrian injuries is performed throughout impact tests using the head, upper leg, and lower leg impactor. (Yong, 2006)

Pedestrian injury simulation is normally performed using the Finite element method at the early design stage to reduce a cost and research period. FE simulation requires detail design data, highperformance equipment and long computation time. FE simulation gives detail results how each part is deformed, how much energy is absorbed and how much injury values are resulted in. But on the other hand, it requires well-designed simulation matrix and many simulations to find contributions to the injury values of various design parameters at the initial design stage.

The system model simulation allows more intuitive parametric studies than the existing detailed FE studies. The computation is much faster than the FE simulation, results are obtained results within in a few seconds and contributions of various parameters are directly get throughout simple parametric simulations.

In this study, the impactor and vehicle system model is developed for the lower leg injury risk assessment. The system model of lower leg impactor, Flex-PLI is developed by comparing to its FE model and system model parameters are calibrated against several static and dynamic certification tests of FLEX-PLI. The vehicle is modeled to equivalent mass-spring-damper systems and its parameters are obtained from existing FE simulation results. And finally developed system model is verified against FE simulation results.
\end{abstract}

Keywords: Pedestrian injury protection, Flex-PLI, System Model

\section{Introduction}

There are many ways of designing cars to improve pedestrian protection. Although the car has many parts and composed with complex sub-structures, the load transmission path of specific impact loading is relatively simple. It is possible to derive important parts for load transfer by analyzing the collision mechanism. And it is possible to construct an intuitive system model for collision problems by calculating equivalent stiffness between major parts.

In this study, system model for lower leg impactor and vehicle is constructed for evaluating lower leg injury. The impactor is based on the latest Flex-PLI (Mallory, 2005). Flex-PLI consists of three parts: Femur, knee, and tibia. Femur consists of 8 segments, tibia has 10 segments and knee consists of four spring wires that represent ACL, PCL, LCL and MCL ligaments respectively. Flex-PLI is covered with multi-layered rubber and pouch cover that expresses the muscles, flesh, and skin of the legs (HUMANETICS, 2011).

The Impactor system model is verified in a variety of ways because it must be able to reproduce injuries that occur on the human body's legs. Static certification test represents the stiffness of the bone structures. Dynamic certification tests such as a pendulum test and an inverse test are performed to verify responses during the impact. In this study, parameters of Flex-PLI subsystems are calibrated using component and static certification tests and are validated using dynamic impact tests.

The vehicle equivalent model was constructed with several equivalent masses that represent set of major parts on the load transmission path and springs and dampers that connect equivalent masses are calculated using FE simulation results. 


\section{System modeling of Impactor system model}

\subsection{Flex-PLI system model}

Flex-PLI has four groups as shown at figure 1: three structural groups (femur, knee, tibia) and one flesh(skin) group. The masses of structures and flesh are $9.38 \mathrm{~kg}$ and $3.82 \mathrm{~kg}$ and total mass of the system is $13.2 \mathrm{~kg}$ (ESI GROUP. FLEX PLI GTR FE Model Users Manual).

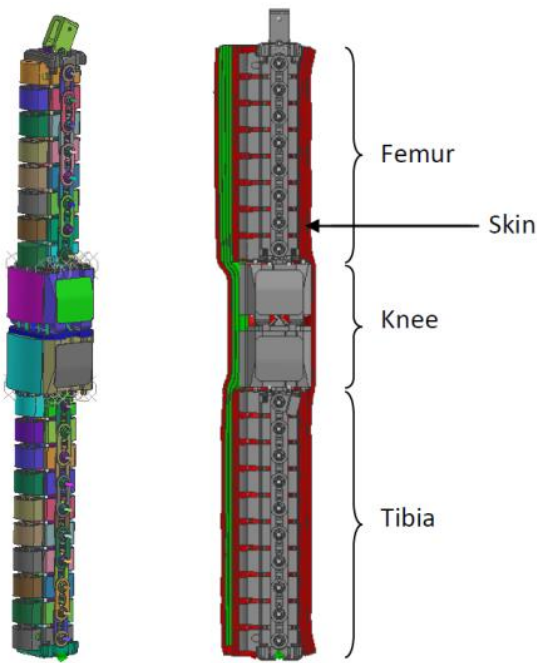

Figure 1. Components of Flex-PLI

\subsubsection{Femur and Tibia assembly model}

Femur and tibia are made of glass fiber reinforced plastic part, take most of the loads and produce bending moment outputs using strain gages which are bonded to it. Femur and tibia are composed of several separated bone segments as shown in figure 2. Each bone segment has a plastic frame, two aluminum spacers, and a cover. Two spacers are rigidly connected to the plastic frame by screws, and the impactor cover is bonded on the frame by a strong double-sided tape. The bone is assembled with the frame by the contact of two spacers. To make sure the spacers are properly contacting the bone, optional thin shims can be used. The link is used to connecting two neighbor bone segments and it maintains uniform space between them. Rubber buffer in the link prevents hard contact between segment. There are four steel wire cables inside the segment corner to prevent a bone damage due to over-bending. Accelerometers are mounted on some segments. (HUMANETICS, 2011)

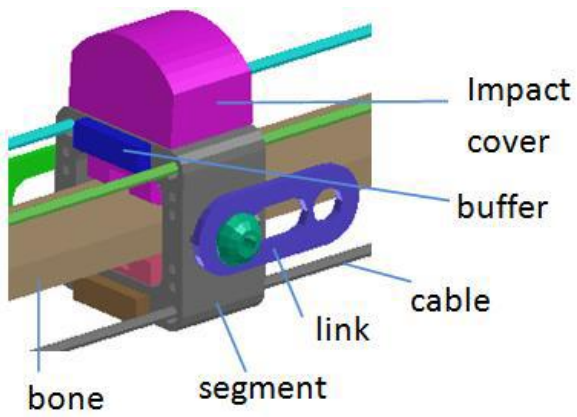

Figure 2. Bone components of Flex-PLI

The system model of bone segments and links are shown at figure 3. Each bone segment is modeled as a rigid body. The link between bone segments is modeled using a revolute joint with rotational spring and damper. The stiffness of rotational springs is calibrated against the static bending certification test as described at section 2.2

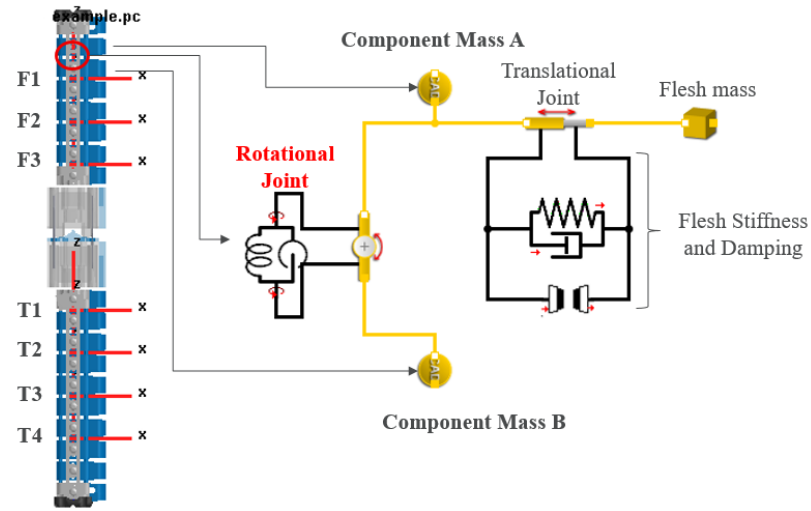

Figure 3. System modeling for femur and tibia

\subsubsection{Knee}

The shape of knee group is shown at Figure 4 without femur block. The knee consists of two aluminum blocks, steel wire, springs, and covers. Two aluminum blocks represent distal femur and proximal tibia and these blocks are contacted each other. Block shapes around contacting area resemble real bone shapes and mimic the real relative motion of bones of human knee. Knee ligaments are represented using steel wires and springs. Springs are designed to meet the required ligament resistive forces and range of motion. They are initially compressed, so the two blocks can hold each other. There is an accelerometer mounted on the lower block and four strings are used to measure MCL, LCL, ACL and PCL elongations. Two impact covers are mounted on the blocks using double-sided tapes. Aluminum covers are used to protect the signal wires and electronics.
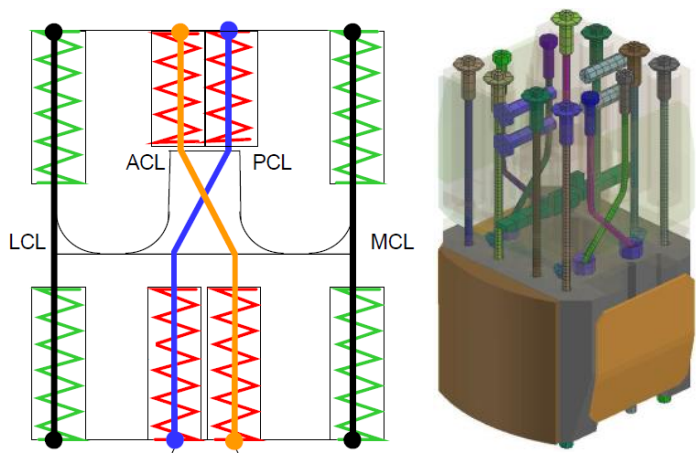

Figure 4. Knee components (source: UN-ECE) 


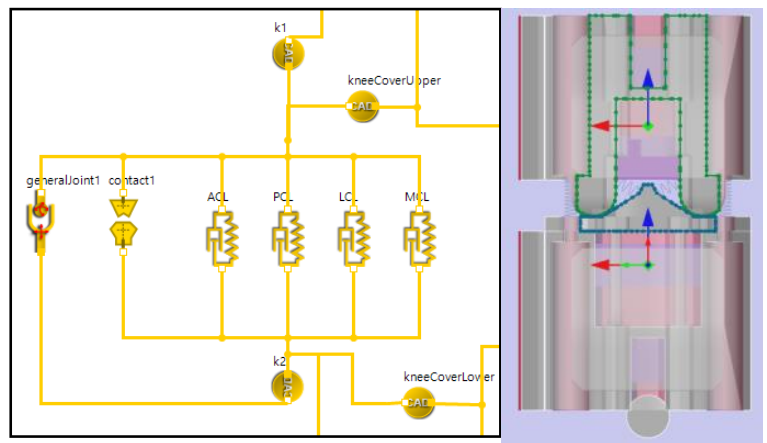

Figure 5. Diagram for Knee assembly and contact

The system model for the knee is shown at figure 5 . Knee blocks are modeled using rigid body and shapes are extracted from FE model. Contact condition is imposed between blocks to represent complicated real relative motions of knee blocks accurately. Four springs connect two blocks and represent knee spring blocks. The stiffness of these springs is calculated using FE model.

\subsection{Flex-PLI static validation}

\subsubsection{Femur and tibia bending simulation}

There is a moment requirement for femur and tibia static bending loading. Maximum moments measured from femur and tibia load cells should be $400 \mathrm{Nm}$ when the specific forces are applied at the center of each assembly. The forces are $4,848 \mathrm{~N}$ and $3,902 \mathrm{~N}$ for the femur and tibia respectively. Static bending simulation using femur and tibia system model is performed to validate the bending stiffness as shown at figure 6 . Simulation results show good correlation as shown at figure 7

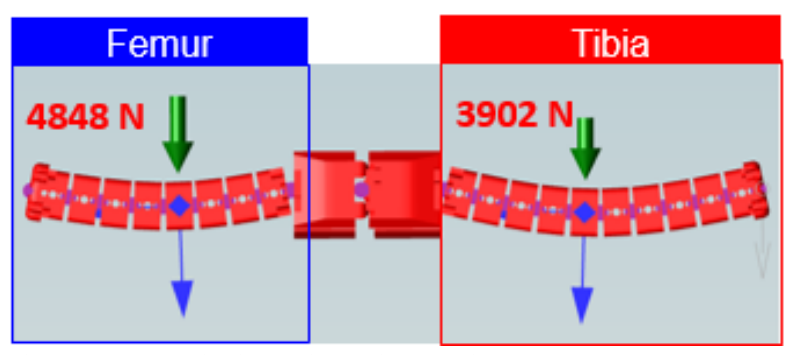

Figure 6. Static bending simulation model

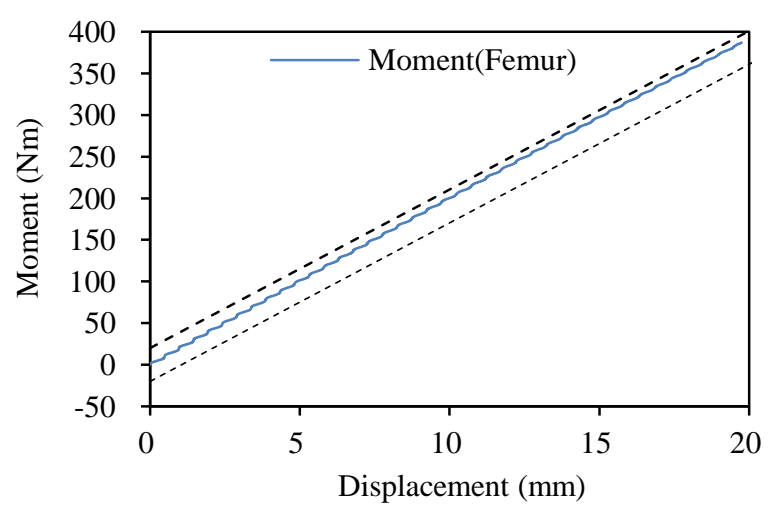

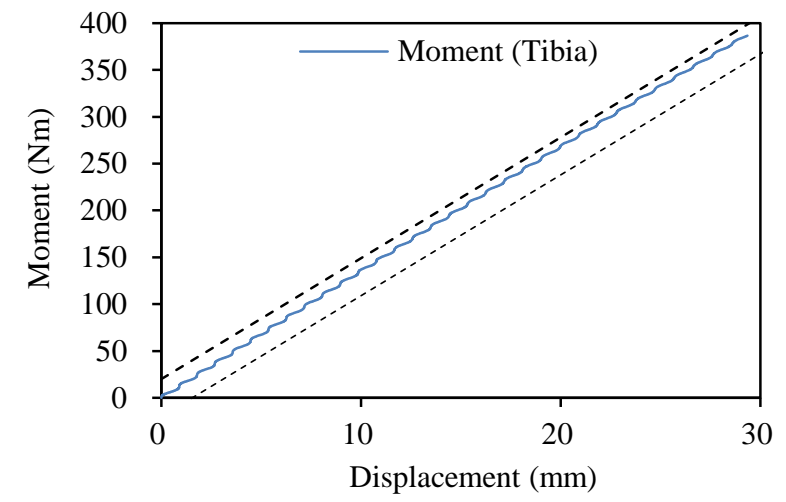

Figure 7. Static bending simulation results of femur and tibia

\subsubsection{Knee bending simulation}

Knee static bending test procedure is shown at figure 8 . As shown at this figure, a round shape of zigs is used at the ends to represent pure bending condition. Force is applied to the proximal tibia end, but round shape loading zig begin to contact the femur right after the loading. The knee static bending simulation is performed using knee system model as shown at figure 9. Simulation results shows good correlation with knee ligament elongation corridors as shown at figure 10.

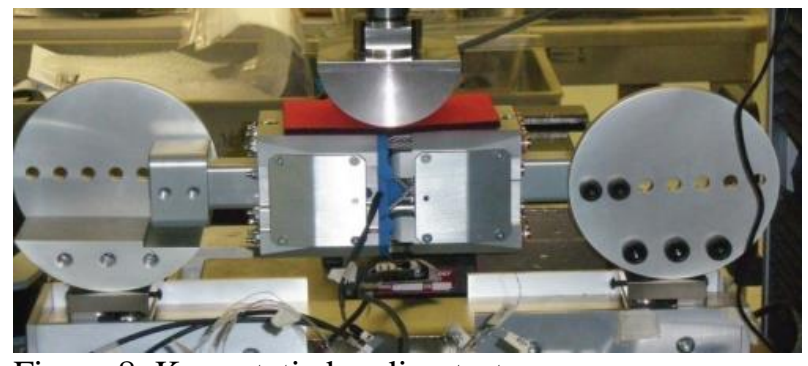

Figure 8 . Knee static bending test

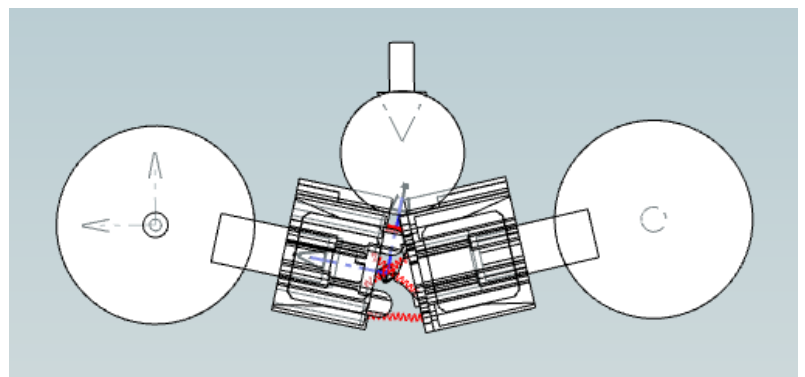

Figure 9. Knee static bending simulation 


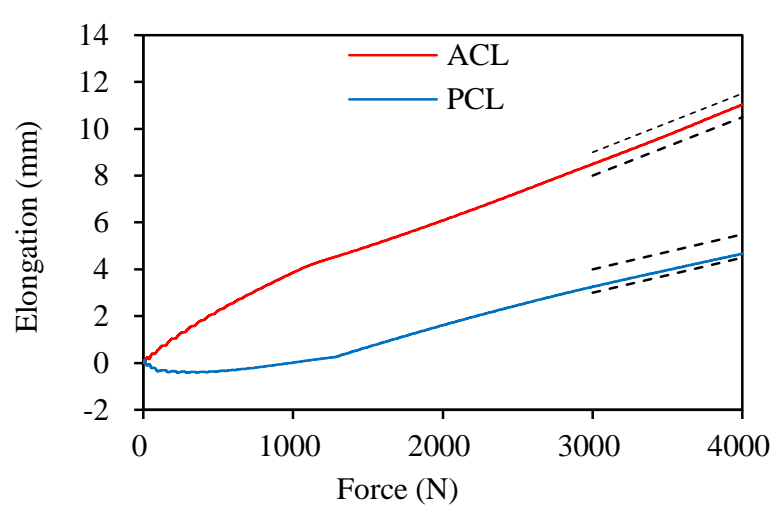

Figure 10. Comparison of knee ligament elongation result for knee static bending simulation

\subsection{Flex-PLI dynamic validation}

In this study, dynamic performance of system model is validated by pendulum and inverse certification tests.

\subsubsection{Pendulum test}

Figure 11 illustrates the pendulum test method. The end of the tibia is linked to the test zig and additional 5 $\mathrm{kg}$ mass installed at the femur end. Before dropping the Flex-PLI, it was lifted by 15 degrees. After it is dropped, the Flex-PLI stopped due to the impact with stopper bar. Moments of femur and tibia and elongations of four ligaments are measured until the Flex-PLI is rebounded.

Pendulum simulation is performed as shown at figure 12 and moment results obtained satisfies corridor as shown at figure 13 .

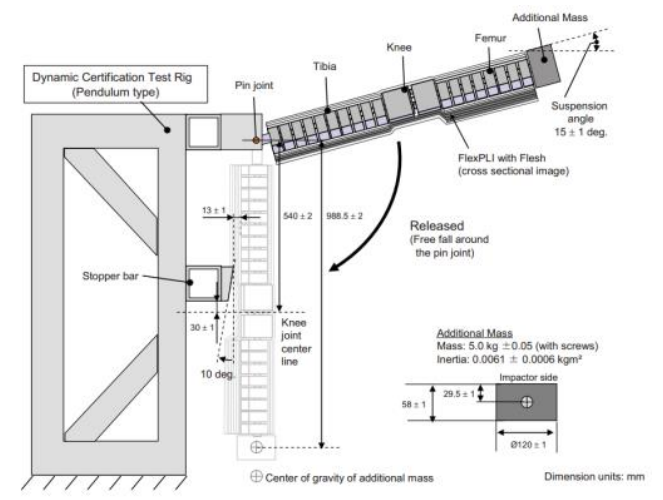

Figure 11. Pendulum test

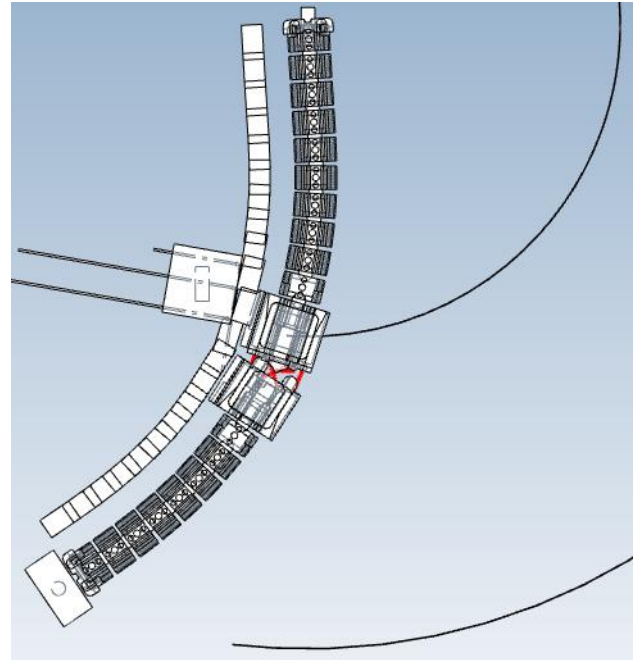

Figure 12. Pendulum simulation model

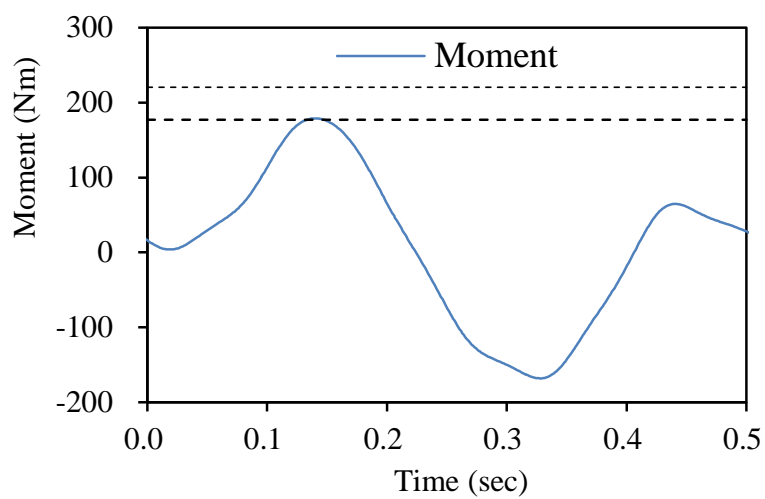

Figure 13. Comparison of moment result for pendulum simulation

\subsubsection{Dynamic inverse test validation}

Figure 14 shows inverse dynamic test. The end of the femur is hanging on the zig, and moving mass impact the lower knee block. The velocity of moving mass is $11 \mathrm{~m} / \mathrm{s}$ and weight is $8 \mathrm{~kg}$. To verify the system model, the inverse test was reproduced as a system model simulation as shown at figure 15 .

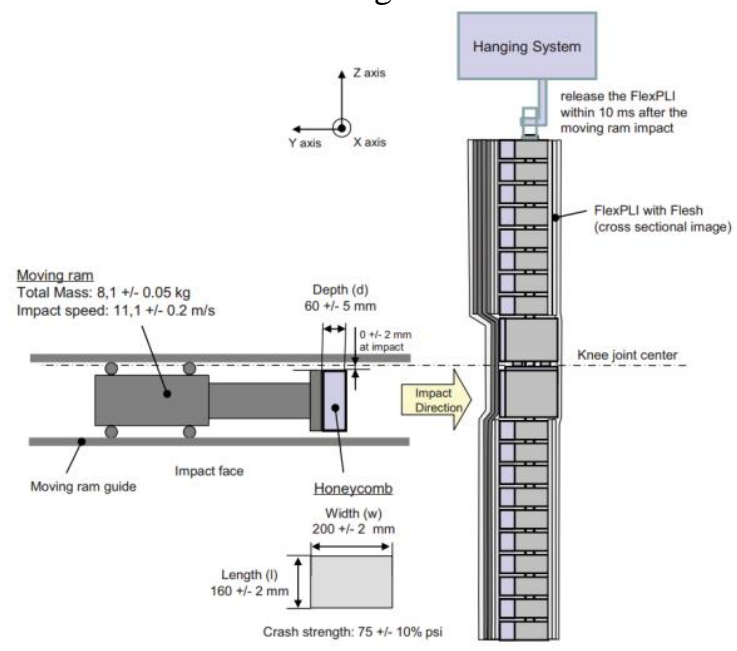

Figure 14. Inverse test 


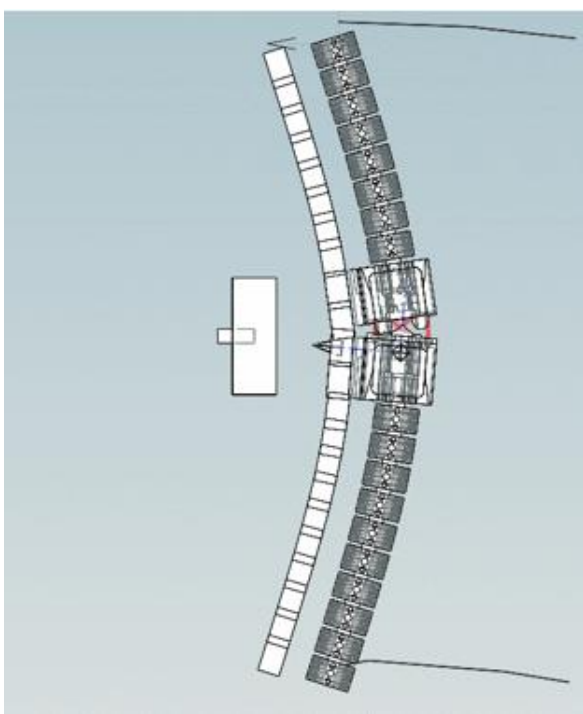

Figure 15. Inverse simulation

Table 1. Comparison of moment for dynamic pendulum simulation

\begin{tabular}{|c|c|c|c|}
\hline Position & test & simulation & Error (\%) \\
\hline F3 & 81 & 52 & $-36 \%$ \\
\hline F2 & 126.2 & 110 & $-13 \%$ \\
\hline F1 & 158.3 & 165 & $4 \%$ \\
\hline T1 & 193.5 & 200 & $3 \%$ \\
\hline T2 & 149.7 & 150 & $0 \%$ \\
\hline T3 & 100.9 & 92 & $-9 \%$ \\
\hline T4 & 47.8 & 40 & $-16 \%$ \\
\hline
\end{tabular}

The end of the femur is restrained and a moving mass of $8 \mathrm{~kg}$ is impacted to the Flex-PLI with $11 \mathrm{~m} / \mathrm{s}$ speed. Contact condition is set between mass and Flex-PLI. In the test, flex-PLI is mounted on the hinge, but it is represented by fixing the corresponding degree of freedom. Table 1 shows the moment results and errors at the measurement positions between tests and system models. Simulation results nearby the knee (T1 and F1) are well matched to test results. Moment errors of femur upper(F3) and tibia lower(T4) are larger and it is mainly because the tensional stiffness of flesh between bone segments are omitted in the system model.

\section{Vehicle parameter extraction from FE model}

The vehicle consists of many parts who transfer forces into directly connected or contacted parts during impact. Due to its complexity, it is almost impossible to extract equivalent stiffness from part results directly. In this study, indirect method is used to extract stiffness and damping parameters between vehicle subsystems using momentum and impulse conservation law via following five steps.

1. Grouping; Flex-PLI impacts to the vehicle fascia. All injury values of Flex-PLI is decided how much reaction forces are generated from the vehicle how much deformation is occurred at the fascia during impact. Thus, vehicle fascia should be divided into several rigid bodies in according to its amount of deformation. During impacting, the impact load is transferred through the hood, upper frontend structure, bumper and lower frontend structures. By analyzing these loading paths, parts included in the same loading path are grouped as a subsystem. In this study, fascia is divided into 7 groups, the hood is divided into 2 groups and engine room structure is divided into 5 groups.

2. Calculation of effective momentum per each group; During impacting, forces are applied into parts, parts move and absorb some energies due to its applied forces and transfer the same amount of forces into other connected or contacted parts. In this study, it is assumed that inertial forces of each group are small because the mass of parts that are majorly involved in the impact is relatively small compared to whole vehicle mass. Then, momentum difference of two groups becomes same as impulse between two groups and impulse becomes same as reaction forces generated from internal energy absorption between two groups. The effective momentum of each group is a summation of all momentums for parts of each group.

3. Calculation of forces between groups; Impulse is calculated from the difference of momentum between two groups. Then, forces are calculated simply by differentiating impulses with time.

4. Calculation of relative displacement between groups; For the fascia, bending behaviors of Flex-PLI are directly decided with fascia frontend displacement which is contacted to Flex-PLI. Thus, for the fascia, frontend displacement of each group is used as a group displacement. For other groups, displacement of group center of gravity is used as a group displacement.

5. Calculation of stiffness between groups; Stiffness is calculated using forces and the relative displacement between groups.

To verify this methodology, simulation is performed using simple bumper impact model as shown at figure 16 and then, stiffness between fascia and back beam is calculated using the proposed method. 


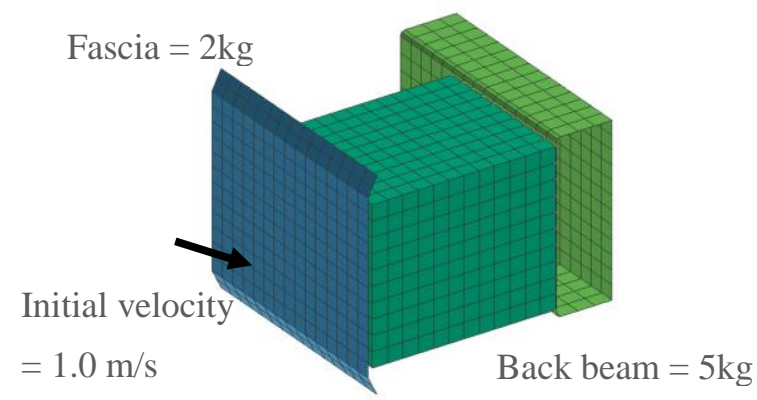

Figure 16. simple bumper model

From the FE simulation results, momentum of fascia and back beam is calculated using part results and momentum difference is obtained as shown at figure 17.

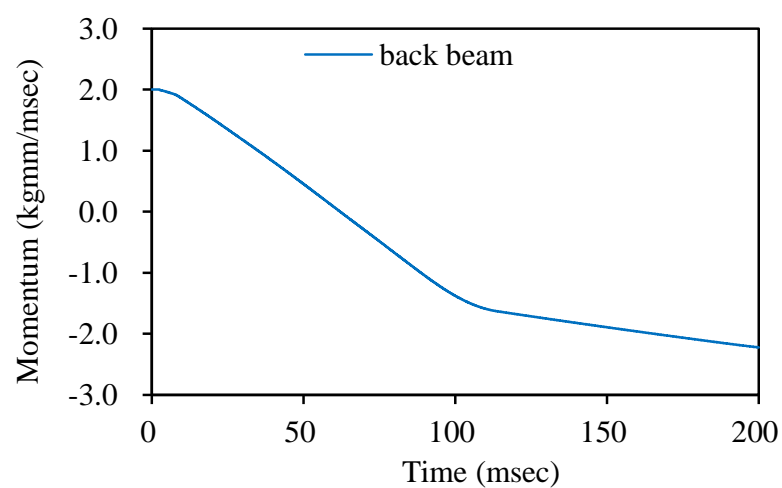

Figure 17. Obtained difference of momentum

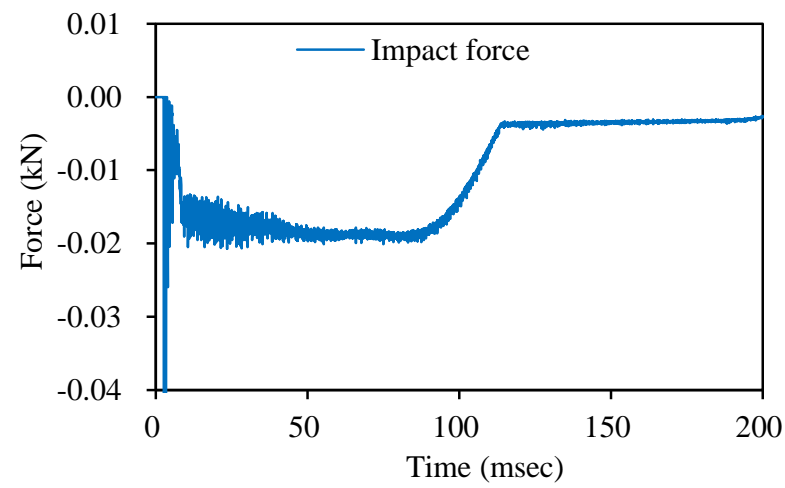

Figure 18. Impact force between fascia and back beam

Momentum difference is same as impulse and forces between fascia and back beam is calculated by differentiating impulse with time as shown at figure 18 . Finally, stiffness between fascia and back beam is calculated using forces and relative displacement of two parts as shown at figure 19 .

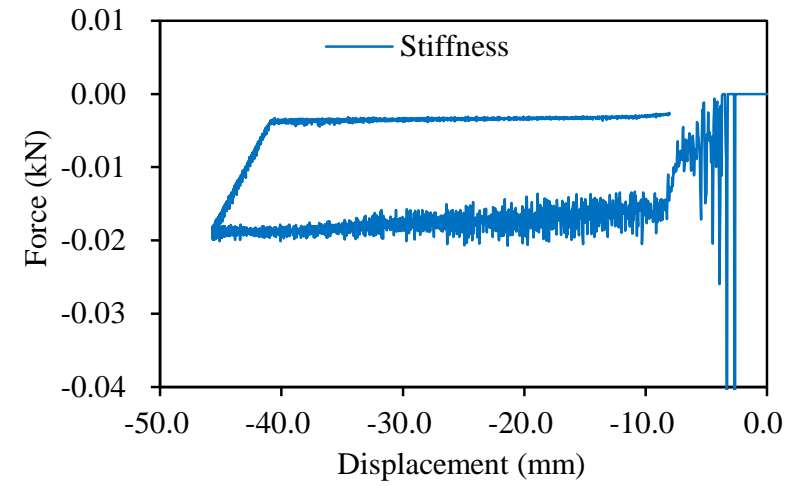

Figure 19. Stiffness between fascia and back beam

To verify stiffness results, the stiffness is converted to engineering stress-engineering strain relationship using foam length and foam area and it is compared with original foam material characteristics.

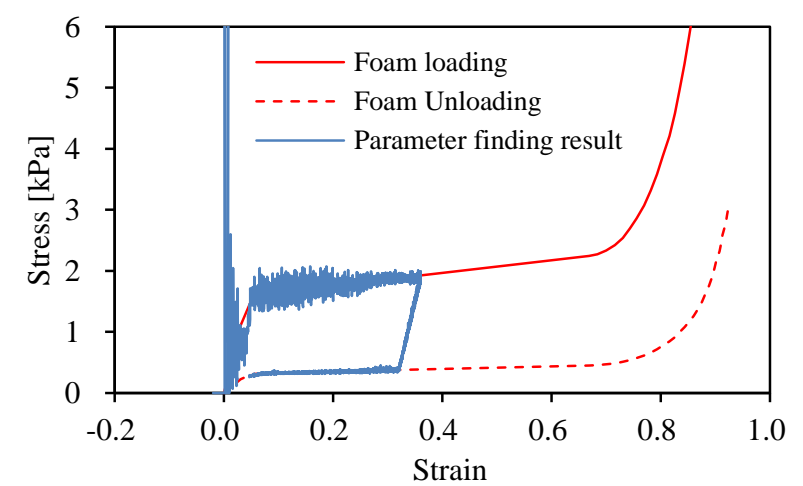

Figure 20. Comparison of stress-strain relationship from stiffness result to original foam material characteristics

As shown at figure 20, stiffness obtained from proposed method will represents internal forces generated between fascia and back beam.

\section{Lower leg impact simulation}

As described in section 3, vehicle system model is composed of 14 groups: 7 fascia groups, 2 hood groups and 5 engine room groups. Vehicle group is the group of parts which are connected to 4 engine room groups and which are not included into other groups.

Each group is modeled using mass. A sliding contact is defined between impactor bone segments and 7 vehicle fascia groups and one hood group and the ground group is entirely fixed. Spring stiffness between groups are calculated using proposed method as described at section 3 and damping coefficient is calibrated to represent unloading behavior of each group.

Figure 22 shows comparison of deformed shape with FE simulation results. As shown at figure 22, when the impactor crashed the vehicle, knee hits the bumper around, femur and tibia ends moved to the vehicle direction till the impactor rebound. And system model 
simulation results are well matched to FE simulation results.

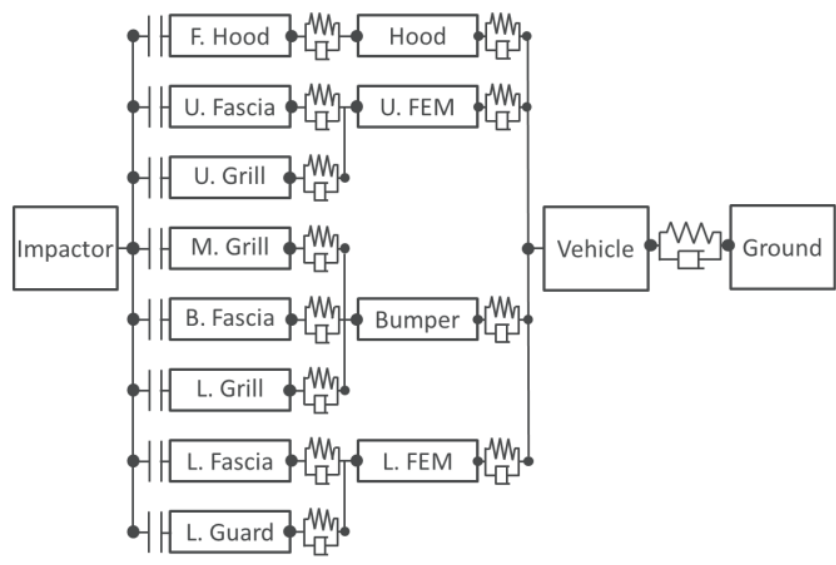

Figure 21. a diagram for the vehicle system model
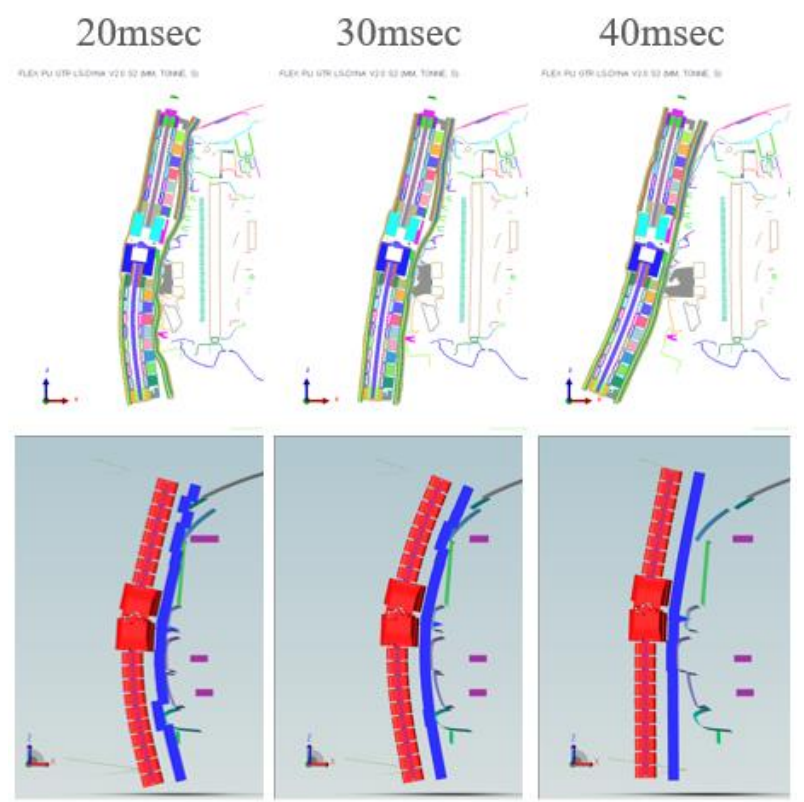

Figure 22. Comparisons between FE and system model

The momentum of bone segments is generated due to different deformation of each fascia groups. Figure $\mathbf{2 3}$ shows the moment of the FE simulation and the system simulation at the main position. The first peak moment occurred at $25 \mathrm{~ms}$. Since the femur and tibia move in opposite directions with respect to the knee, the directions of moments are opposite to each other and the impactor is rebounded after $25 \mathrm{~ms}$. In the case of the System model, the behavior up to the first peak was very similar to the FE results, but there was a difference after the impactor rebound. This is because the springs in the vehicle model express loading phase well but are not sufficient to express the stiffness in unloading phase. However, since the maximum damage caused by the collision occurs mostly in the first peak, the prediction of the injury is possible from the results of this study.
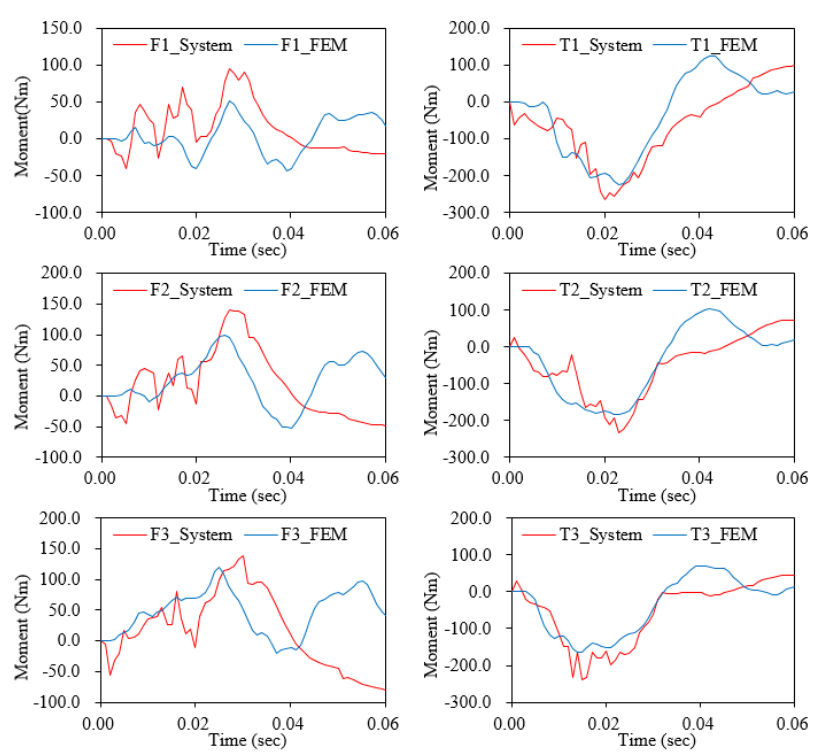

Figure 23. Compare moments between FE simulation and system simulation

\section{Conclusion}

In this study, a system model is constructed to analyze the pedestrian lower leg impact. The system model for lower leg impactor, Flex-PLI, is constructed and verified against various static and dynamic certification tests. Methodology for constructing vehicle system model is proposed based on momentum and impulse conservation law and it is illustrated using simple bumper impact model. Vehicle system model is finally constructed by grouping all vehicle parts into 14 groups, calculating stiffness between groups using the proposed method. Finally, whole lower leg impact simulation model is simulated and verified by comparing its results with FE simulation results. The impactor moment results of FE simulation and the system simulation are well matched in terms of the magnitude of the moment and the peak time. In the case of system simulation, calculation time is completed within 5 minutes in the single core machine, so it is very efficient to review various parameters at the initial stage of design.

\section{References}

HUMANETICS. Flex PLI GTR User Manual, 2011

ESI GROUP. FLEX PLI GTR FE Model Users Manual,

ESI GROUP. ITI SimulationX User Manual 3.8

J A Carroll, A Barrow, B J Hardy, B Robinson, Transport Research Laboratory, Pedestrian legform test area assessment report, 2014

Ann Mallory, Jason A. Stammen, France Legault, 19th International technical conference on the Enhanced Safety of Vehicles. Component leg testing of vehicle front structures, 05.0194, 2005 
Boo-Joong Yong, Hyun-Deog Cho, Jae-Wan Lee, Journal of the Korean Society of Manufacturing Process Engineers. Development of vehicle evaluation system for pedestrian protection, Vol. 5 No. 4, pp.53 58, 2006 